\title{
Gradhiva
}

GRADHI

Revue d'anthropologie et d'histoire des arts

$7 \mid 2008$

Le possédé spectaculaire

\section{Le caboclo surmoderne}

Globalisation, possession et théâtre dans un temple d'umbanda à Fortaleza (Brésil)

The supermodern caboclo. Globalisation, possession and theatre in an Umbanda temple in Fortaleza (Brazil)

\section{Erwan Dianteill}

\section{(2) OpenEdition}

\section{Journals}

Édition électronique

URL : http://journals.openedition.org/gradhiva/999

DOI : $10.4000 /$ gradhiva.999

ISSN : 1760-849X

Éditeur

Musée du quai Branly Jacques Chirac

Édition imprimée

Date de publication : 15 mai 2008

Pagination : 24-37

ISBN : 978-2-915133-86-8

ISSN : 0764-8928

Référence électronique

Erwan Dianteill, « Le caboclo surmoderne », Gradhiva [En ligne], 7 | 2008, mis en ligne le 15 mai 2011, consulté le 02 mai 2019. URL : http://journals.openedition.org/gradhiva/999 ; DOI : 10.4000/ gradhiva.999 


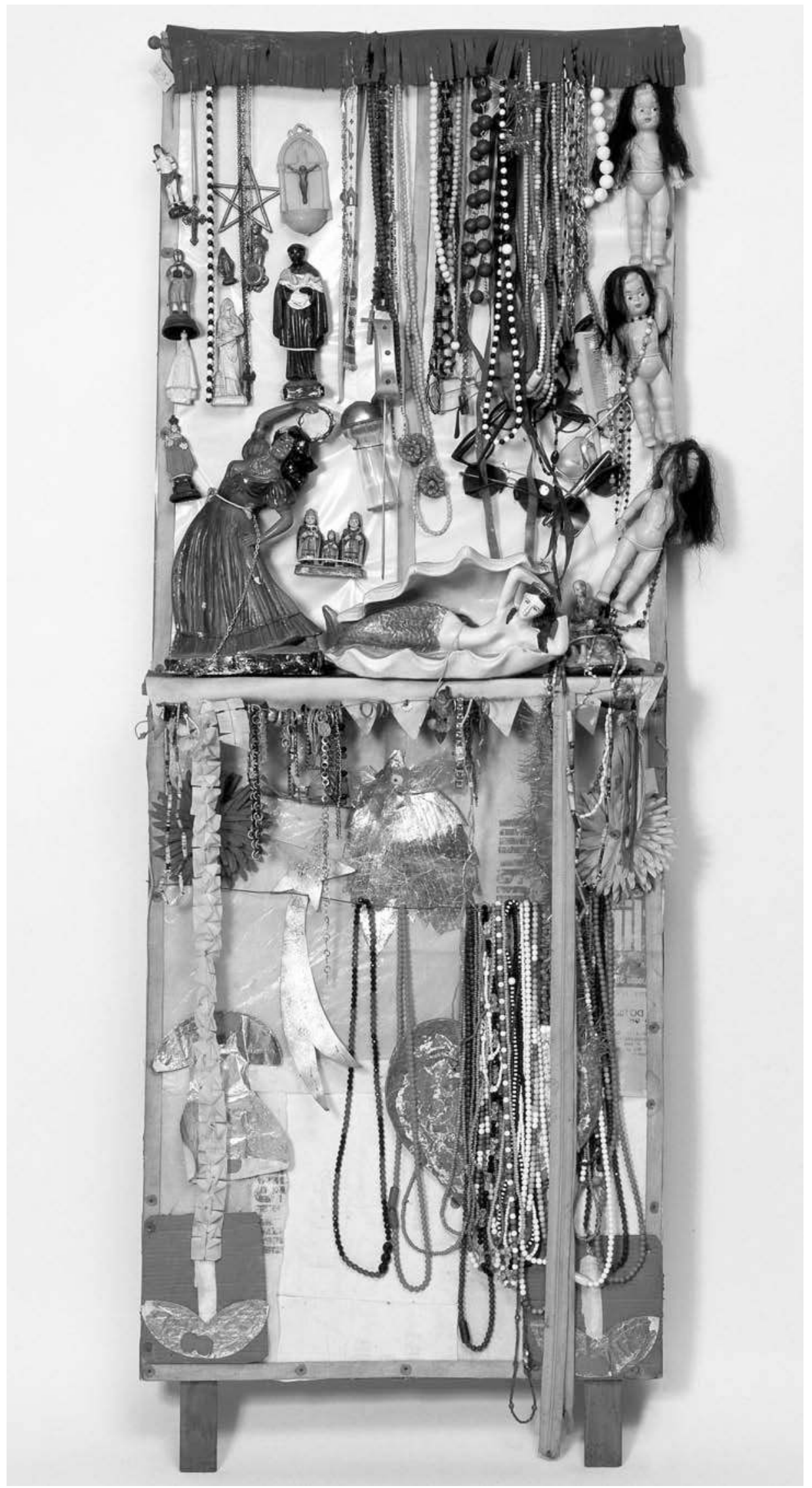

Fig. 1 Macumba de Arthur Bispo do Rosario (1911-1989). Installation. Métal, plâtre, plastique et carton. Coleção Museu Bispo do Rosário. IMAS Juliano Moreira, Rio de Janeiro. @ Photo Fernando Chaves. 


\title{
Le caboclo surmoderne
}

\author{
Globalisation, possession et théâtre dans un temple d'umbanda \\ à Fortaleza (Brésil)
}

Erwan Dianteill

S

i la majorité des études sur la globalisation reposent sur la dialectique entre le local et le global, les anthropologues doivent prendre en compte une autre dimension lorsqu'ils s'intéressent à la religion : les mouvements d'ascension et de descente des esprits, déplacements qui jouent un rôle décisif dans la liturgie. Toutes les religions (ou presque) conçoivent la relation avec les êtres surnaturels en leur assignant un lieu de résidence - le ciel, les grottes, le fond des lacs ou la cime des arbres. La hiérarchie des esprits correspond souvent à une hiérarchie spatiale, du dieu suprême résidant dans les nuées aux esprits infernaux. Nous savons bien sûr que cette hiérarchie n'est pas universelle, mais varie en fonction des cultures considérées. L'important reste que l'étude de la globalisation religieuse, définie comme la circulation généralisée des agents, biens et services religieux, passe par la prise en compte des représentations spatiales et cosmologiques des croyants, qui orientent les flux, les implantations, les déplacements. En d'autres termes, on comprendra peut-être la globalisation économique en observant le transport des marchandises d'un point à un autre de la surface du globe, mais il nous faut examiner la façon dont les croyants conçoivent la relation entre le monde humain et l'empire des esprits pour saisir la globalisation religieuse. Cette idée sera illustrée par des éléments provenant de mes propres observations de terrain et de sources secon- daires relatives à l'umbanda, une religion née au Brésil d'influences en partie africaines. On trouvera en premier lieu une présentation rapide de l'histoire de l'umbanda, focalisée sur les processus de déterritorialisation et de reterritorialisation ayant affecté l'Afrique, puis le nord et le sud du Brésil. En second lieu, il s'agira de décrire les formes prises par l'umbanda dans le Ceará, État du Nordeste brésilien où la population d'origine africaine est très minoritaire. La dernière section sera consacrée à l'analyse d'une cérémonie observée dans un temple de la périphérie de Fortaleza ${ }^{1}$, cérémonie dans laquelle l'articulation du local et du global dans la possession apparait pleinement.

\section{Umbanda, ou comment être Africain sans être Africain}

Selon Roberto Motta, «s'il fallait définir l'umbanda en peu de mots, on dirait qu'elle consiste dans l'application d'une théologie, dérivée du spiritisme européen "codifié" par Alan Kardec (nom de plume de Léon Rivail, 1804-1869), aux croyances et aux pratiques afro-brésiliennes traditionnelles, sous la forme de la macumba,

1. Je remercie chaleureusement Ismael Pordeus, professeur d'anthropologie à l'université fédérale du Ceará, qui m'a introduit dans le milieu de l'umbanda à Fortaleza, et dont les ouvrages dans ce domaine [2000, 2002) m'ont été extrêmement utiles. 
typique de la région de Rio " (2002:115). On peut tenter d'aller un peu au-delà de cette brève définition en s'intéressant à l'origine historique et géographique de cette religion. Bastide (1960 : 422-475), Ortiz (1978), Brown et Bick (1987), Giobellina Brumana et Gonzalez Martinez (1989), ainsi que Prandi (1995 : 68-73) donnent des informations convergentes sur le contexte social de sa naissance. Il s'agit, dès son origine, d'une religion globalisée, au sens défini plus haut.

Selon ces auteurs, l'umbanda a été créée à Niteroi, près de Rio de Janeiro, à la fin des années 1920 par un groupe de Brésiliens blancs des classes moyennes pratiquant le spiritisme kardeciste. Ces gens avaient en outre une bonne connaissance des religions d'origine africaine, acquise au contact des descendants d'esclaves au Brésil. Ils connaissaient principalement le candomblé de Salvador de Bahia, sous ses différentes formes, et la macumba de Rio, un ensemble cultuel moins organisé que le candomblé. La macumba et le candomblé étaient profondément enracinés dans le prolétariat noir, tandis que le catholicisme était la religion des élites blanches. L'umbanda trouvait donc un terrain propice à son développement dans les classes moyennes du sud du pays. Les rites, les croyances, l'ethos et l'organisation sociale de l'umbanda sont en relation avec la position de ce groupe dans la structure sociale du Brésil de cette époque.

La dissidence des umbandistes par rapport au spiritisme s'explique par le rejet des esprits indiens (caboclos) et noirs (pretos velhos ${ }^{2}$ ) par les kardecistes, qui les considèrent comme des entités inférieures dans la hiérarchie des esprits. Les umbandistes acceptent l'idée que ces esprits n'ont pas atteint un haut niveau de pureté, et c'est précisément pour cette raison qu'ils les ont placés au cœur du rituel. Conçus comme des entités proches des hommes, caboclos et pretos velhos peuvent agir plus aisément en leur faveur que des esprits plus lointains. En outre, l'intégration de l'Indien et du Noir était un symbole de "brésilianité ». Le catholicisme était associé au Portugal, ancienne puissance coloniale, et le candomblé à l'Afrique des esclaves. L'umbanda se veut au contraire une religion moderne et nationale, qui synthétise les sources européenne, africaine et indienne, en affinité avec le régime républicain proclamé en 1889. C'est une des raisons pour lesquelles une partie importante des premiers umbandistes étaient des fonctionnaires fédéraux de Rio. Ces employés de bureau, soldats, maîtres d'école ou avocats partageaient une même croyance dans le progrès porté par le nouvel État brésilien.
Pour autant, tous les éléments de la synthèse umbandiste ne se voient pas accorder une même dignité. Certaines pratiques africaines, comme le sacrifice animal, sont disqualifiées car perçues comme primitives. Dans l'évolutionnisme kardeciste dont hérite l'umbanda, on considère que les religions les plus avancées sont les plus détachées de la matière, et du sang en particulier. En tant que religion la plus nouvelle et la plus spirituelle, l'umbanda ne peut donc accepter le sacrifice animal.

Les umbandistes ont créé une religion dont la relation avec l'Afrique s'est faite de plus en plus distante. D'abord, au niveau rituel, le yoruba, le kikongo ou le fonewe ont cessé d'être les langages de communication avec les esprits. Depuis les années 1930, les cantiques sont chantés en portugais, et beaucoup d'entre eux sont dérivés des hymnes spirites et même de la musique populaire. Les Brésiliens blancs et les nouveaux immigrants européens ne maîtrisaient pas les langues africaines en usage dans le candomblé du Nordeste. Les rythmes et les danses du candomblé ont été simplifiés, tout comme les phases de l'initiation. D'une certaine façon, l'umbanda est une religion plus démocratique que le candomblé. L'initiation est plus courte, moins coûteuse (on économise le prix des animaux). La possession devient une expérience beaucoup plus courante, elle n'est plus réservée aux initiés.

En ce qui concerne les croyances, la configuration et la hiérarchie du " panthéon " des dieux yoruba ont été réorganisées. Le nombre de divinités (orixas) du candomblé est réduit, et elles sont devenues des forces abstraites et transcendantes, qui résident dans l' « astral " sans descendre sur terre. Pour communiquer avec les humains, les orixas leur envoient des esprits messagers. Ils sont conçus comme les chefs des «lignes », ou "phalanges ", d'esprits inférieurs. Du point de vue éthique, l'umbanda adopte une position intermédiaire entre catholicisme et candomblé. Dans cette dernière religion, les entités ne sont ni «bonnes " ni « mauvaises ", tout dépend de la relation entretenue avec elles, et en particulier des offrandes qu'on leur fait, du respect ou de la transgression des interdits qu'elles imposent. Les divinités provoquent des troubles et peuvent aussi y remédier. Quant au catholicisme, il repose sur une partition radicale entre bonnes entités - Dieu, les anges et les saints - et mauvaises entités - le diable et ses serviteurs.

2. Pour une analyse des sens sociopolitiques multiples du preto velho, voir Hale 199 ? 


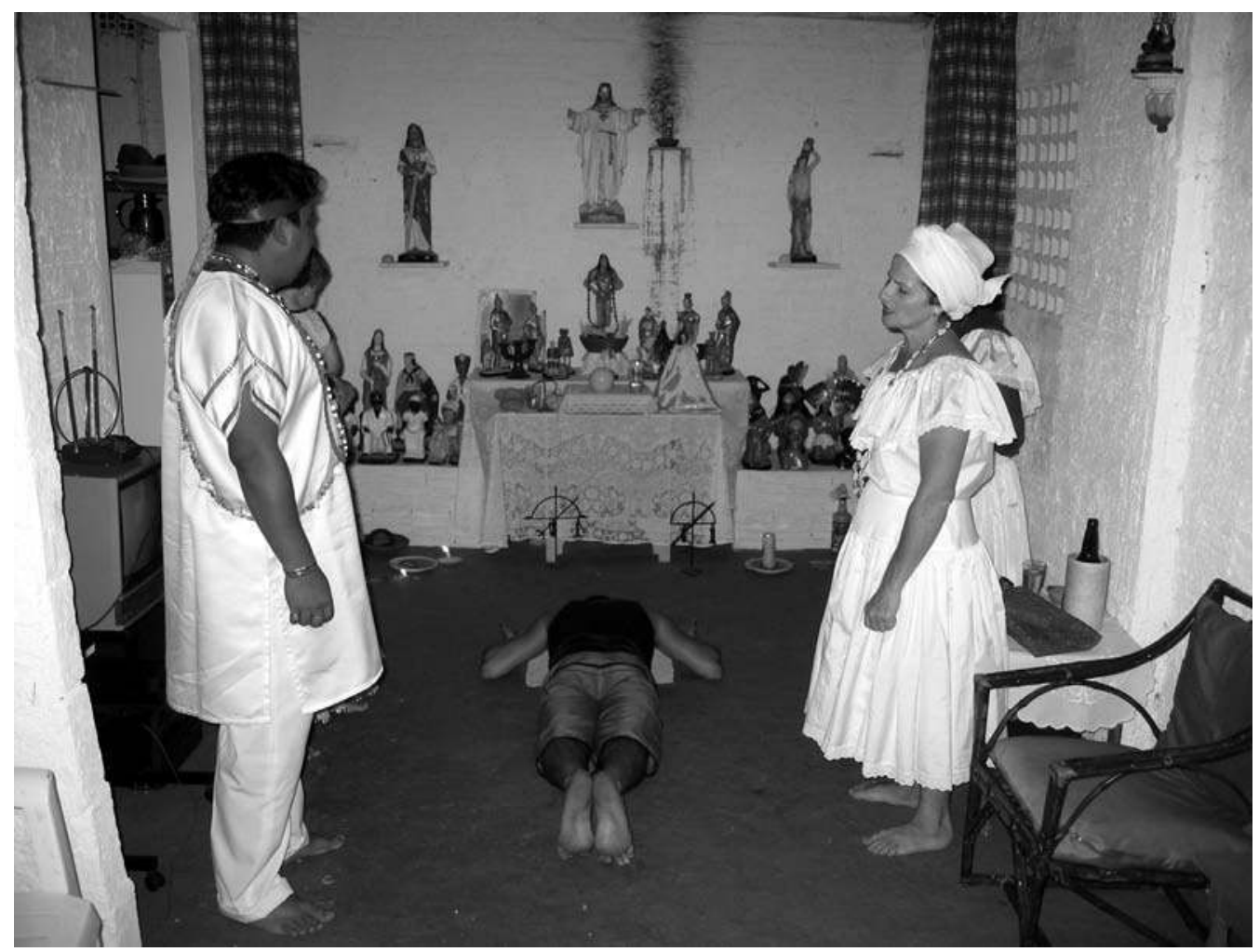

Fig. 2 Prière au caboclo pai Roxo. Fortaleza, 2004. Photo Erwan Dianteill.

L'éthique umbandiste n'est ni catholique ni africaine, ce qui n'empêche pas une distinction entre deux types d'action. Dans la première, appelée linha da direita, le côté droit, on a affaire à des entités «éclairées » : cette voie est celle de l'umbanda stricto sensu. Les orixas sont à la tête de ces entités : les vieux Noirs (pretos velhos) et les Indiens (caboclos ${ }^{3}$ ), et aussi des vachers (boaideros), des esprits-enfants (crianças) ou des marins (marinheiros). Du côté "gauche ", a linha da esquerda, on traite avec des esprits obscurs, demeurés aux premiers stades de l'évolution spirituelle : c'est la voie de la quimbanda. On y entretient des relations avec les esprits d'anciennes prostituées (pombas giras), de gitanes (ciganas) ou de souteneurs (exus). Ce n'est pas un hasard s'il s'agit des groupes les plus marginaux et les plus dangereux de la société brésilienne. Leur pouvoir provient précisément de leur capacité à refuser les règles communes en matière de sexualité, de violence, d'argent. Ce sont des rebelles à l'ordre social imposé par les classes dominantes, des "maîtres du désordre " selon l'expression de Bertrand Hell (1999). À la différence du catholicisme, qui prohibe absolument la fréquentation des forces infernales, l'umbanda maintient les deux voies, liées organiquement. On peut travailler avec les esprits de la droite ou de la gauche, en fonction des circonstances.
En d'autres termes, le côté gauche est la contrepartie rituelle du côté droit, il n'est nullement interdit et il est même intensivement pratiqué dans les temples (terreiros). Comme l'écrivent Fernando Giobellina Brumana et Elda Gonzalez Martinez (1989 : 182-183) :

La droite est un type de manipulation qui : a) est seulement fait en relation avec le client, b) a été demandé par lui, et c) poursuit une finalité dont la légitimité ne peut être mise en question du point de vue des valeurs dominantes. La gauche, en plus du fait que ce type d'action peut agir au-delà du client, a) impose son action à une tierce personne, b) contre sa volonté, c) avec une finalité dont la légitimité - toujours d'un point de vue officiel extérieur - est douteuse ou inexistante [...]. L'évaluation du commerce avec le côté gauche n'est pas de nature morale mais plutôt pragmatique. Ce qui compte, ce sont les avantages et désavantages que de telles ressources impliquent.

L'umbanda représente une solution à la contradiction entre le " pur " pragmatisme des religions africaines autochtones toujours en vigueur dans le candomblé et la morale chrétienne : la relation avec les esprits y

3. Le caboclo est à l'origine un homme de l'intérieur du Brésil, Indien ou métis d'Européen; dans l'umbanda, certains esprits de caboclos sont d'origine africaine. 


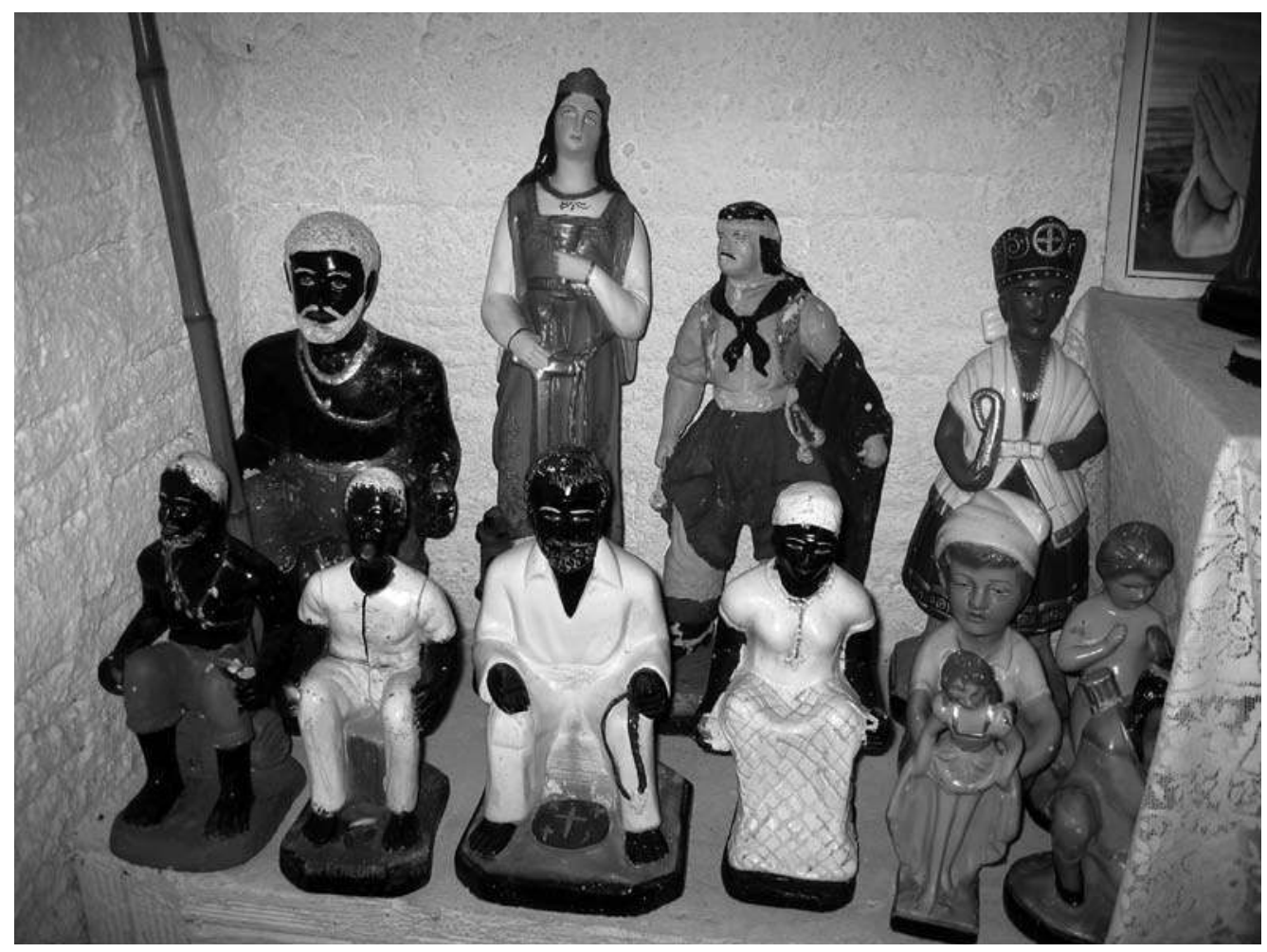

Fig. 3 Autel des pretos velhos. Fortaleza, 2004. Photo Erwan Dianteill.

est pragmatique, puisque les pratiquants poursuivent leur propre intérêt, mais ce rapport inclut de façon non morale la moralité d'origine catholique. Le cœur de l'umbanda (et de la quimbanda) est donc cette relation composite avec des esprits capables d'intervenir dans l'existence humaine (maladie, argent, amour, problèmes judiciaires).

Notons aussi que les umbandistes ont construit un nouveau mythe d'origine. Depuis sa fondation au Brésil, la provenance africaine du candomblé ne fait pas de doute : il a toujours été reconnu comme une religion implantée au Brésil par les esclaves ${ }^{4}$. À Salvador de Bahia, les trois principales traditions du candomblé se rapportent chacune à un groupe culturel africain : les Yoruba (appelés Nagô ou Ketu), les Kongo/Mbundu (appelés Angola) et les Ewe/Fon (nommés Jêjê). En outre, la mémoire collective des pratiquants transmet le nom du fondateur et de sa nação (son groupe africain d'origine), un lexique de cantiques et des mythes dont la source ne fait aucun doute. Même si des personnes blanches ou mulâtres sont initiées dans le candomblé, nul ne nie que la structure rituelle, mythique et organisationnelle de cette religion trouve sa source en Afrique. Or, le fait que les orixas sont venus d'Afrique et que le mot même umband $a^{5}$ ait une consonance bantoue était en contra- diction avec l'idée que l'umbanda était la dernière religion à apparaître, donc la plus « avancée » : comment les esprits les plus importants de l'umbanda peuventils être originaires de l'une des régions les moins « civilisées » du globe ? La solution trouvée par les théoriciens de l'umbanda fut de considérer l'Afrique comme un simple espace de transition. Lors du premier congrès de spiritisme d'umbanda, qui se tint à Rio de Janeiro en 1941, des représentants approuvèrent l'idée que leur religion trouvait sa source en Lémurie ${ }^{6}$ (Bastide 1960 : 446). D'autres umbandistes estimaient que leur religion venait d'Égypte ancienne, d'autres encore de l'Inde ancienne, umbanda étant considéré comme un mot sanskrit. Tous ne voyaient dans les pratiques africaines qu'une dégénérescence de ces traditions anciennes, et leur renaissance brésilienne comme une régénération. En bref,

4. Pour une bibliographie complète sur le candomblé, voir le catalogue de l'exposition au musée Dapper (Paris), Brésil, l'héritage africain (Falgayrette-Leveau et Dianteill 2005).

5. Notons que le mot kimbànda, qui se réfère à la voie gauche dans le vocabulaire de l'umbanda, signifie «jalousie » en kikongo (lari) (Nsondé 1999 : 101).

6. Depuis la seconde moitié du XIX ${ }^{\mathrm{e}}$ siècle, la Lémurie appartient au corpus des mythes de l'occultisme occidental; on la considère comme un continent englouti, un équivalent oriental de l'Atlantis. 
comme l'écrivent Brown et Bick (1987: 80), « les premiers leaders de l'umbanda manifestaient une extrême ambivalence à l'égard de l'Afrique, acceptant la contribution culturelle africaine d'un côté, cherchant à la désafricaniser d'un autre côté" .

On peut ainsi résumer l'organisation symbolique de l'umbanda par une série d'oppositions à la fois symboliques et spatiales:
Ogum. Il a dit : «Écoute-moi, va enregistrer ce temple en tant que centre de spiritisme d'umbanda São Jorge, écoute attentivement... ", et il l'a dit trois fois, je l'ai entendu.

En d'autres termes, l'umbanda du Ceará n'est pas une religion autochtone comme le candomblé de Salvador (ce qui ne l'a pas empêchée de s'y développer avec succès ${ }^{7}$ ). La légitimité des leaders, en tout cas des premiers d'entre eux, vient de leur initiation à Rio ou

\begin{tabular}{|l|c|c|c|c|c|c|c|c|c|}
\hline Umbanda & haut & blanc & pur & bon & orixas & ciel & Orient & Sud du Brésil & moderne \\
\hline Quimbanda & bas & noir & impur & mauvais & exus & rues & Afrique & Nordeste & archaïque \\
\hline
\end{tabular}

Le niveau inférieur n'est nullement exclu dans ce système. Il en est au contraire constitutif : il existe une intense circulation sociale, spatiale et symbolique entre les deux paliers. Cette idée est illustrée ici par le cas d'un temple d'umbanda à Fortaleza, capitale de l'État du Ceará.

\section{Le temple de pai Roxo à Fortaleza}

Au Ceará, l'umbanda est une religion importée ; elle inclut, comme l'umbanda du sud du pays, un grand nombre de rituels et de croyances africaines, sans pour autant que sa base sociale soit «noire ». En effet, l'économie de plantation et l'esclavage n'ont jamais été dominants dans le Ceará. La population étant majoritairement d'origine européenne et amérindienne, les religions d'origine africaine n'ont pas été implantées directement dans la région. Après la fin de l'esclavage au Brésil (1886), les migrations internes ont entraîné une dissémination des religions afro-brésiliennes : des personnes originaires du Ceará ont migré et ont été initiées à Recife, Salvador de Bahia, São Paulo ou Rio de Janeiro, puis certaines sont revenues dans leur région d'origine. La fédération d'umbanda du Ceará a été fondée en 1954 (Pordeus 2002 : 12), soit treize ans après le premier congrès d'umbanda en 1941 à Rio. Voici la façon dont Mãe Julia, la fondatrice du premier temple d'umbanda du Ceará, relatait en 1978 l'enregistrement officiel de l'institution qu'elle dirigeait (ibid. : 106) :

Vous savez que l'umbanda n'existait pas ici au Ceará, c'était quelque chose d'affreux, de caché, c'était comme ça... Eh bien, quand je suis allée à Rio de Janeiro, j'ai passé un an dans un centre [d'umbanda], avec ma mère-de-saint, et quand j'étais là-bas, mon rêve [meu ideal] était : " Ah! Quand je vais rentrer au Ceará... " Quand je suis rentrée, après trois ou quatre jours, j'ai prié, et j'ai reçu un ordre de mon chef, c'est-à-dire l'orixa
São Paulo, où l'umbanda naquit. L'umbanda de Fortaleza est donc une dérivation de l'umbanda originale, ellemême en partie dérivée du candomblé du Nordeste. Ce processus illustre bien les migrations religieuses internes dans le pays.

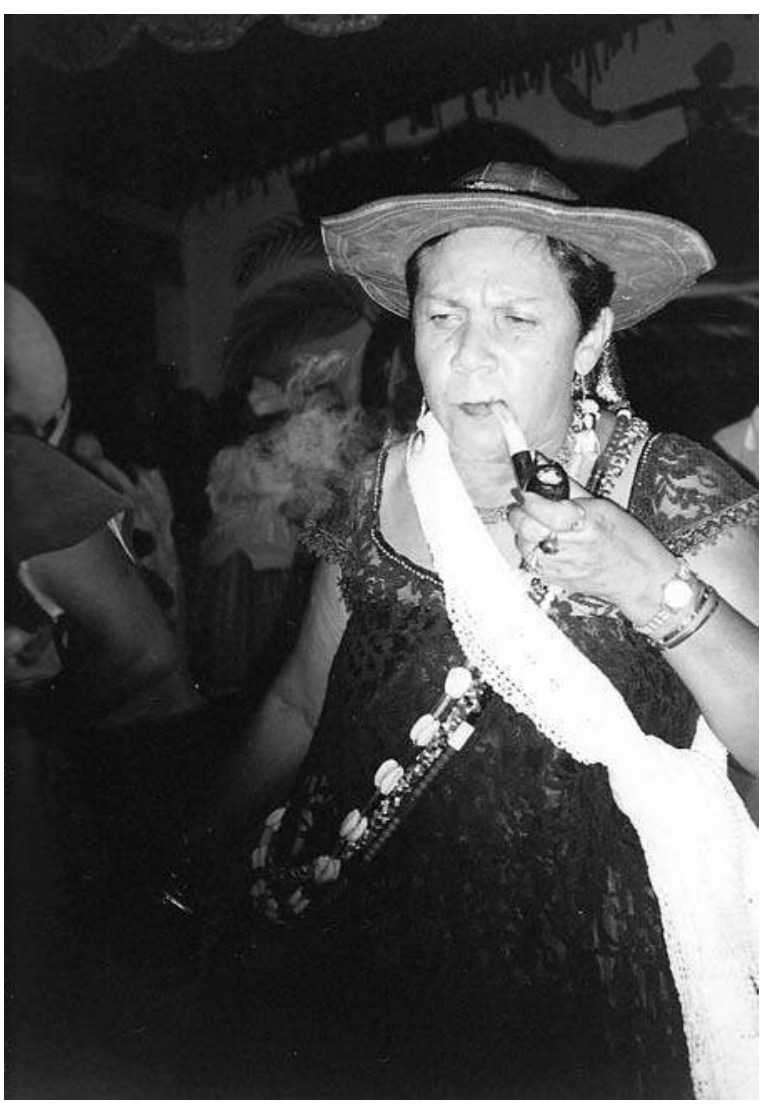

Fig. 4 Fille-de-saint possédée par un esprit boiadero. Fortaleza, 2005. Photo Ismael Pordeus (I. P.)

7. En 1977, Jean Duvignaud consacre une part importante de l'ouvrage Le Don du rien à des cérémonies d'umbanda à Fortaleza. À cette époque, la fête de lemanjá, au mois d'août, attirait selon lui deux à trois mille personnes sur la plage du Futur [2007 [1977] : 36]. 
En 2002 et en 2004, j'ai visité quatre temples d'umbanda lors d'une enquête de quelques semaines à Recife ${ }^{8}$ et Fortaleza. Mon propos portera seulement sur l'un d'entre eux, le terreiro de pai Roxo. C'est un tout petit temple, situé dans un quartier périphérique de la ville, sans appartenance à aucune fédération locale ou nationale de temples umbandistes. Il est particulièrement intéressant car il n'est soumis à aucune norme institutionnelle. Il a été fondé par João ${ }^{9}$ dans la ferme familiale (fazenda), dans la périphérie rurale de la ville, à environ vingt kilomètres au sud-est du centre-ville. Cet homme a aujourd'hui environ 65 ans. Il a travaillé comme gardien d'immeuble à Manaus, puis à São Paulo. Tout comme Mãe Julia, il fut initié à l'umbanda dans cette ville. Le détour par Manaus, sur lequel il insista dans la conversation informelle que j'eus avec lui, a un autre sens : c'est là qu'il entra en contact avec les caboclos, car Manaus est la grande ville d'Amazonie. C'est une importante source de légitimité car le caboclo Roxo, l'esprit éponyme du temple, est un caboclo de la forêt : c'est un indien chasseur de la tribu Indaia (é de tribo de Indaia), selon un cantique d'umbanda.

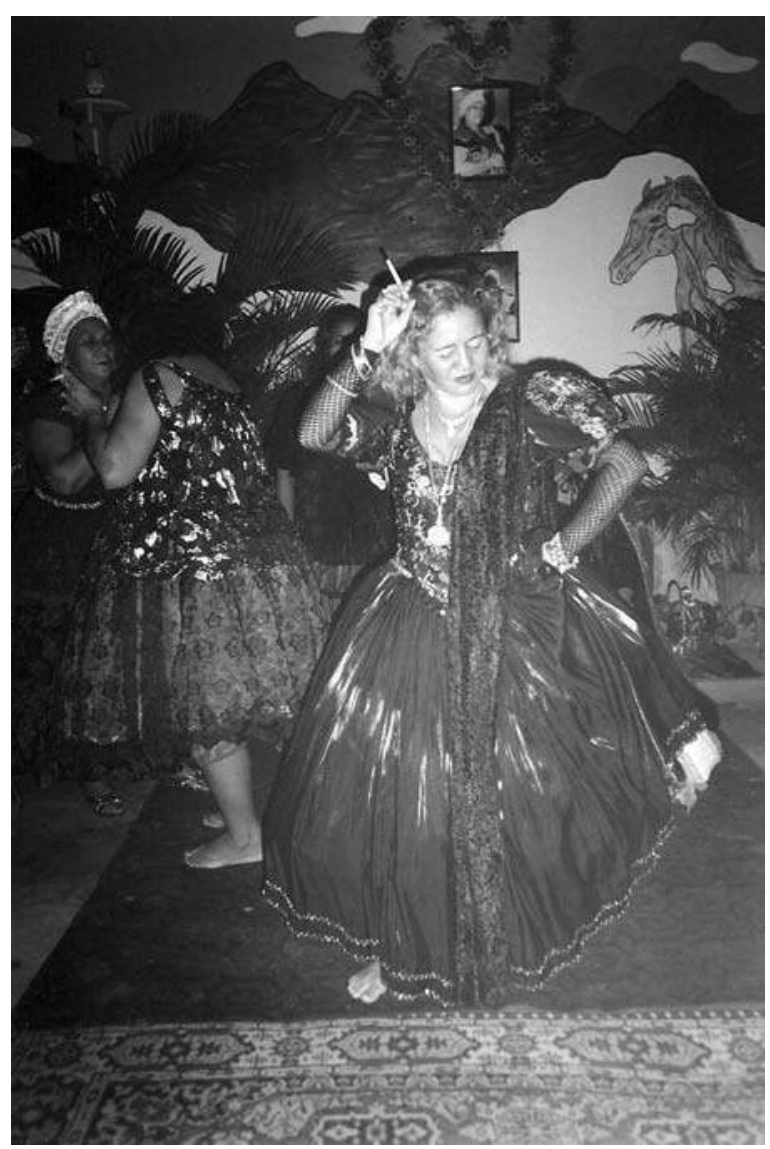

Fig. 5 Mère-de-saint possédée par une pomba gira. Fortaleza, 2005. Photo I. P.
La capacité de João à guérir, qui occupe en général une place importante dans l'umbanda mais peut-être plus encore dans ce temple, est associée à un autre événement. João a (selon sa sœur) été infecté par le virus du sida à la fin des années 1980 et s'est arrêté de travailler à cette époque. Il est rentré vivre à Fortaleza, où il a créé son terreiro au début des années 1990. Depuis lors, le caboclo Roxo prend soin de lui, soulage sa maladie et l'utilise pour soigner d'autres malades. Selon João, le nombre de membres a culminé à environ soixante personnes, puis il a dû fermer le temple au milieu des années 1990, à cause de «mauvaises influences ». On trouve en effet dans l'umbanda l'idée que des visiteurs peuvent venir accompagnés de mauvais esprits charriant l'envie, la haine, la folie. Plus le nombre de membres d'un temple est important, plus ce risque est grand. Lorsque j'ai visité le temple en 2004, l'assistance était réduite à une douzaine de personnes.

Le temple, de plain-pied, est localisé dans l'un des trois bâtiments qui composent la ferme; entre ces bâtisses, des animaux (chiens, poules, chèvres) sont en liberté. Une cuisine et un vestiaire communiquent avec la salle principale, un rectangle de dix mètres sur quatre. À une extrémité de celle-ci, à droite de la porte d'entrée, se trouvent deux tambours mono-membranaires. Les participants à la cérémonie s'assoient sur des chaises en plastique, dos aux tambours et face à l'autel situé à l'autre extrémité de la pièce, sur deux niveaux. Trois statues sont fixées au mur, en hauteur : à gauche une statue de sainte Barbe, au centre une statue de Jésus vêtu d'une robe blanche et à droite une statue de saint Sébastien percé de flèches.

Sous chacune de ces statues, une table soutient un ensemble d'autres représentations. Sous l'image de sainte Barbe, qui est aussi associée à Yansan, l'orixa de la tempête, on trouve un groupe de statuettes de Vieux Noirs (pretos velhos); sous saint Sébastien, associé à l'orixa Oxossi, divinité de la forêt et de la chasse, on distingue un ensemble de statuettes d'Indiens, les caboclos; sous la statue de Jésus, qui représente aussi Obatala, dieu de la paix, reposent des effigies d'entités à la peau blanche : une statue de centurion représentant saint Georges ou, près d'un lion, un vieillard figurant saint Jérôme. Ces images d'esprits subalternes, placés en position basse, dépendent des esprits supérieurs qui les dominent. Habituellement, ce sont les esprits de personnes ayant vécu sur terre, et qui sont devenues les

8. Je remercie Roberto Motta pour son aide précieuse à Recife.

9. Pseudonyme. 
serviteurs, les messagers des orixas après leur mort. Les exus constituent une classe d'esprits distincts des précédents. Ils ne sont pas représentés sur l'autel de João; il est possible qu'un autre autel, moins public, leur ait été consacré à l'extérieur du temple, mais João ne me l'a pas montré.

La classification ternaire, c'est-à-dire blanc/noir/ indien, est manifeste dans la structure de cet autel; elle l'est aussi dans les couleurs utilisées pour décorer le temple. Le sol est de couleur verte, une bande jaune d'un mètre de large court le long du mur (l'encadrement des fenêtres est également vert), le reste des murs et le plafond sont blancs. Juste en face de l'autel, un carré blanc de vingt-cinq centimètres de côté environ est peint sur le sol vert. Le fundamento d'Oxala est enterré sous ce carré, au-dessus duquel pend un récipient circulaire rempli d'eau bénite. Le bleu, le jaune et le vert font partie des couleurs nationales brésiliennes, ce qui correspond bien à l'idée que l'umbanda est la religion brésilienne par excellence. Le blanc est associé à la population d'origine européenne et le vert aux Indiens de la forêt, mais on comprend moins bien l'usage du jaune dans ce contexte. En fait, cette couleur est celle de Xango-saint Jérôme dans l'umbanda alors que, dans le candomblé, la couleur de Xango est le rouge, tout comme dans la santeria cubaine (voir Dianteill 2000). On peut supposer que le noir et le rouge sont exclus des couleurs officielles du temple car ils sont associés à Exu, l'esprit du mauvais garçon dans l'umbanda, et par extension à la violence (rouge) et la sorcellerie (noir). Le jaune de Xango (et non le rouge) serait une euphémisation des potentialités dangereuses des pratiques de la quimbanda. L'usage du jaune permettrait ainsi la construction d'une homologie entre les esprits, les gens et les couleurs nationales.

En résumé, l'espace du temple de pai Roxo est fortement structuré symboliquement (haut/bas, disposition des statues, usage des couleurs) et socialement (Européen, Africain, Indien). Les dieux résident dans l'« astral " tandis que les esprits subalternes descendent sur la terre et interagissent avec les êtres humains.

\section{Interactions habituelles ${ }^{10}$}

J'ai été conduit au temple de pai $^{11}$ Roxo par Maria ${ }^{12}$, la sœur du chef de culte, qui est bibliothécaire à Fortaleza. Samedi 6 novembre 2004, j'ai été invité pour la première fois à une cérémonie dédiée au pai Roxo. Jusquelà, je n'avais eu que des contacts téléphoniques avec le chef de culte, le " père-de-saint ». Pendant la cérémonie, trois types d'agents se sont côtoyés dans le temple.

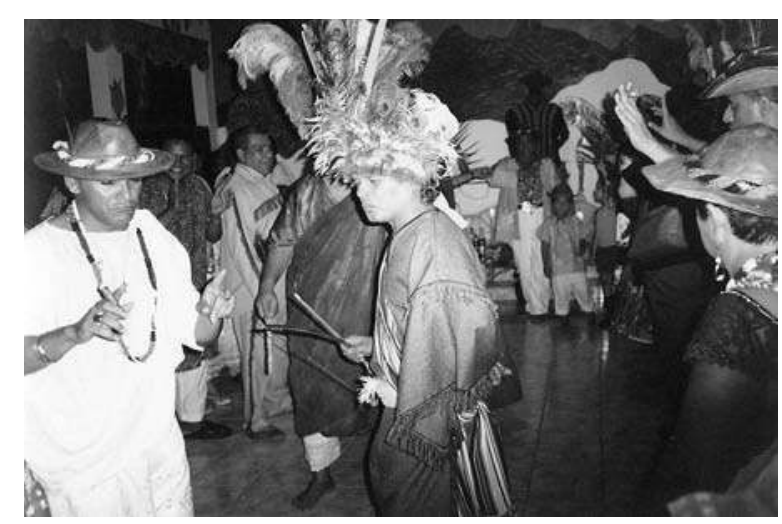

Fig. 6 Fils-de-saint lors d'une cérémonie de boiadero. Fortaleza, 2005. Photo I. P.

D'abord, des visiteurs, membres ou non du terreiro, entraient ou sortaient de la pièce principale. Ce groupe comprenait une quinzaine de personnes. Certains étaient vêtus de blanc; Maria était habillée en bahiana, avec une longue robe blanche couvrant plusieurs jupons et un foulard blanc sur la tête. J'ai collecté peu d'informations sur ces personnes, mais elles semblaient appartenir aux classes populaires des faubourgs de Fortaleza. La majorité d'entre elles avaient entre 20 et 50 ans, avec un nombre sensiblement égal d'hommes et de femmes. Le cœur de la cérémonie consistait en une consultation personnelle avec un esprit incarné dans le corps d'un médium, et si nécessaire en un « travail ${ }^{13}$ » spirituel réalisé sur-le-champ. Ainsi, une femme participait pour la première fois à une cérémonie dans ce temple et venait avec une collègue de bureau. Les médiums lui demandèrent de se tenir dans un cercle qu'ils formèrent en se tenant la main, puis ils la firent tourner sur elle-même jusqu'à ce qu'elle vacille, retenue par une assistante non possédée, et qu'elle se mette à pleurer : il s'agissait d'expulser un mauvais esprit qui la troublait. C'est une cérémonie très fréquente dans l'umbanda, adaptée du kardecisme et nommée desobsessão (Hess 1989, 1990).

En deuxième lieu, plusieurs médiums étaient possédés par des entités. Lorsque je suis arrivé, trois esprits étaient présents. Le premier était le caboclo Roxo luimême, que João, le père-de-saint, avait incorporé. Le nom Roxo provient d'un type de bois violet tirant sur le jaune, le pau-roxo do Amazonas; ce nom indique que ce cabo-

10. Je qualifie ces interactions $d^{\prime} \ll$ habituelles 》 car elles ne diffèrent pas de celles que j'ai pu observer dans d'autres temples d'umbanda.

11. Le terme pai [«père 》) peut aussi bien désigner le chef de culte (pai João) que l'esprit (pai Roxo) ; il marque le respect.

\section{Pseudonyme.}

13. Sur le sens du mot trabalho dans la macumba de Rio, équivalent à celui qu'il prend dans l'umbanda, voir Pordeus 2000 : 67-120. 
clo est bien un esprit de la forêt. Selon João, interrogé après la fin de la cérémonie, c'est l'esprit d'un vieil Indien plein de sagesse ; il aime fumer des cigarettes et boire de la bière quand il descend sur terre. On le connaît dans de nombreux terreiros, mais dans celui de João, seul le père-de-saint peut l'incorporer. Le caboclo Roxo, lorsqu'il possédait le corps de João, clignait des yeux de façon ininterrompue, de sorte qu'il était impossible de croiser son regard. Une femme noire d'une cinquantaine d'années, habillée comme Maria, écrivait sur un cahier les recommandations du pai Roxo car, lorsque les esprits abandonnent la terre, les médiums ne peuvent en aucun cas se rappeler ce qu'ils ont dit puisqu'ils ne sont pas les locuteurs de ces énoncés. Il faut donc un secrétaire pour noter les recommandations du caboclo, le consultant étant souvent trop impliqué dans l'interaction pour s'en souvenir. Selon le caboclo lui-même (on verra plus loin comment il s'en est expliqué), son travail sur terre est surtout thérapeutique : il soigne les gens. Le sacrifice animal étant interdit dans le temple, pai Roxo ne travaille qu'avec des bougies, du miel et du parfum pour libérer ses "enfants » (c'est-à-dire les membres du temple) des mauvaises influences. Lorsqu'il vivait sur terre, c'était une personne mauvaise, puis il est mort et il monta vers le « karma " où il fut purifié, avant d'être renvoyé sur terre par Jésus ${ }^{14}$ pour faire le bien.

Selon Maria, pai Roxo est le chef de tous les esprits du temple, ce qui n'empêche pas d'autres esprits de descendre, de posséder un médium, de consulter et de soigner les gens. Parmi ces esprits, on trouvait ce soir-là des ogums (c'est-à-dire des messagers de l'orixa Ogum, dieu du fer), des pretos velhos, des pombas giras (littéralement « colombes tournoyantes ") et des ciganas (gitanes), qui sont des esprits d'anciennes prostituées, ainsi que des caboclos avec des personnalités distinctes de celle du pai Roxo. Dans ce temple, comme dans tous les terreiros d'umbanda, il est habituel qu'un médium incorpore successivement plusieurs entités lors d'une même cérémonie ${ }^{15}$. La sortie d'une entité et l'entrée d'une autre dans un corps humain sont parfois instantanées, mais il y a souvent une courte "crise " entre ces deux moments : un cri, une contraction musculaire, un déséquilibre.

Enfin, des agents distants appelaient constamment par téléphone pour consulter les esprits. Certaines de ces personnes habitaient la ville; d'autres téléphonaient de beaucoup plus loin, du sud du pays, de Rio en particulier. À ces catégories d'acteurs « classiques » dans un terreiro (hormis peut-être les consultants à distance), il faut en ajouter une : celle de l'anthropologue étranger. Nous allons voir que sa présence peut générer des effets inattendus.

\section{Interactions inhabituelles}

J'avais parlé à João par téléphone, mais celui-ci étant possédé par son caboclo quand je suis arrivé au temple, il ne pouvait évidemment pas me le présenter. C'est donc Maria qui m'a permis d'engager la conversation avec pai Roxo. Celui-ci parlait un portugais altéré, Maria traduisait pour moi ce qu'il disait. Je lui ai alors posé des questions volontairement naïves : qui était-il ? D'où venait-il? Dans quel but? Ce genre de dialogue avec une entité n'est pas commun : en général, les gens consultent les esprits pour des affaires privées, et non pour débattre de questions théologiques. Pai Roxo ne semblait pas très assuré dans ses réponses, au moins au début de la discussion. Quand je lui ai demandé s'il y avait un diable et un enfer, par exemple, il commença par me répondre que rien de tel n'existait, que le mal venait seulement de l'homme et que les méchants vivent déjà en enfer sur terre. Les esprits ne sont pas responsables du mal qu'ils font, ce sont les gens qui envoient les esprits faire le mal qui le sont. Puis, quand je l'interrogeais sur les «travaux » qu'il faisait pour les gens, il me répondit qu'il fallait lutter contre les mauvaises influences de certains esprits, les anges rebelles. Son discours passait donc d'une conception du mal comme fait humain à une conception surnaturelle du mal causé par les anges déchus issue de la théologie catholique. Néanmoins, après quelques questions, l'entretien se transforma en leçon collective : le caboclo se tourna vers les membres qui attendaient leur consultation pour leur dire d'écouter attentivement, car selon lui peu d'entre eux connaissaient la religion d'umbanda. L'interview devint une sorte de cours où pai Roxo instruisait les membres, certains paraissant un peu étonnés de la tournure que prenait la cérémonie.

Cet étonnement se mua en franc divertissement quand pai Roxo décida de quitter la terre pour laisser place à un autre caboclo du nom de Simbamba. Ce dernier est un esprit grossier et farceur, dont toutes les phrases sont émaillées de saillies grivoises et de jurons (elles commençaient par Puta que pariu!), le tout accom-

14. Lors de la conversation que j'ai eue avec lui, pai Roxo se référait fréquemment à Jésus, mais pas à la Bible. Le karma est une notion dérivée de l'hindouisme, ici synonyme de «séjour des âmes ».

15. La capacité à incorporer successivement une série d'entités diverses lors d'une seule cérémonie distingue l'umbanda du candomblé, en tout cas dans sa version nagô (yoruba), ainsi que de la santeria cubaine: dans ces deux derniers cas, on ne peut incorporer qu'une seule entité, qui doit être un orixa. La possession par des entités inférieures peut avoir lieu, mais dans un cadre rituel distinct; à Cuba, il peut s'agir d'une messe de spiritisme (voir la lettre de Bastide à Leiris à ce sujet : 68-69). 
pagné de gestes obscènes suscitant l'hilarité générale. L'essentiel de son discours consistait à se vanter de la taille de son pénis et du grand nombre de femmes avec lesquelles il avait fait l'amour sans jamais être fatigué. Quand il prit la place de pai Roxo dans le corps de João, j'étais toujours face à lui; l'entretien commencé avec pai Roxo sur un ton moraliste et didactique prit donc un tour de bouffonnerie incontrôlable. Simbamba se servit de moi pour faire rire le public. Son premier conseil était de me faire proférer des jurons - ce dont, selon lui, j'avais le plus besoin car «c'est excellent pour la santé ». Il proclama qu'il allait me donner une leçon de portugais gratuite, et il me fit effectivement répéter après lui les pires obscénités, ce qui encouragea encore les gens à rire dans la salle. D'autant plus que, me prêtant au jeu, je demandais la signification des mots. Simbamba priait alors Maria, un peu gênée par la tournure prise par la conversation, de l'aider à me faire comprendre le sens des expressions. La joie du public fut à son comble lorsque Simbamba prit un téléphone qui sonnait et se trouva en ligne avec une femme de Rio de Janeiro, à qui il déclara : «Bonjour! Tu ne sais pas la dernière, j'apprends le portugais à un Français en ce moment... Écoute, tu vas voir si je mens. » Le caboclo me tendit le combiné et je me retrouvais à débiter comme lui des obscénités à une personne stupéfaite, à trois mille kilomètres de là. Le moins que je puisse dire est que le public appréciait ce numéro : on se moquait d'un étranger, plus précisément d'un Français, supposé être particulièrement raffiné, et par la même occasion d'un habitant de Rio, que l'on imagine toujours un peu hautain à l'égard des habitants du Nordeste. Simbamba remplissait parfaitement son rôle de trickster en inversant toutes les valeurs de l'umbanda défendues dix minutes plus tôt par pai Roxo. Le discours de Simbamba était ainsi une sorte de leçon "à l'envers ». Alors que pai Roxo visait à édifier les membres du terreiro, Simbamba fit exactement le contraire, singeant même la posture de maître d'école du premier.

À la fin du service religieux, après le départ de Simbamba, un autre esprit transgressif posséda João. Il $\mathrm{s}^{\prime}$ agissait de la cigana, la gitane ${ }^{16}$. Lorsqu'elle descendit sur lui, João quitta la salle principale pour s'habiller dans les « coulisses », c'est-à-dire dans le vestiaire attenant à la cuisine, avec une assistante. Après une demiheure, la cigana revint habillée d'une robe de scène écarlate couverte de sequins, les pieds dans des chaussures à talons aiguilles, parée de bijoux. Elle utilisait en outre un éventail espagnol pour se rafraîchir, mais aussi pour cacher son visage. Maria m'expliqua que la gitane ne pouvait supporter d'avoir incorporé un visage aussi

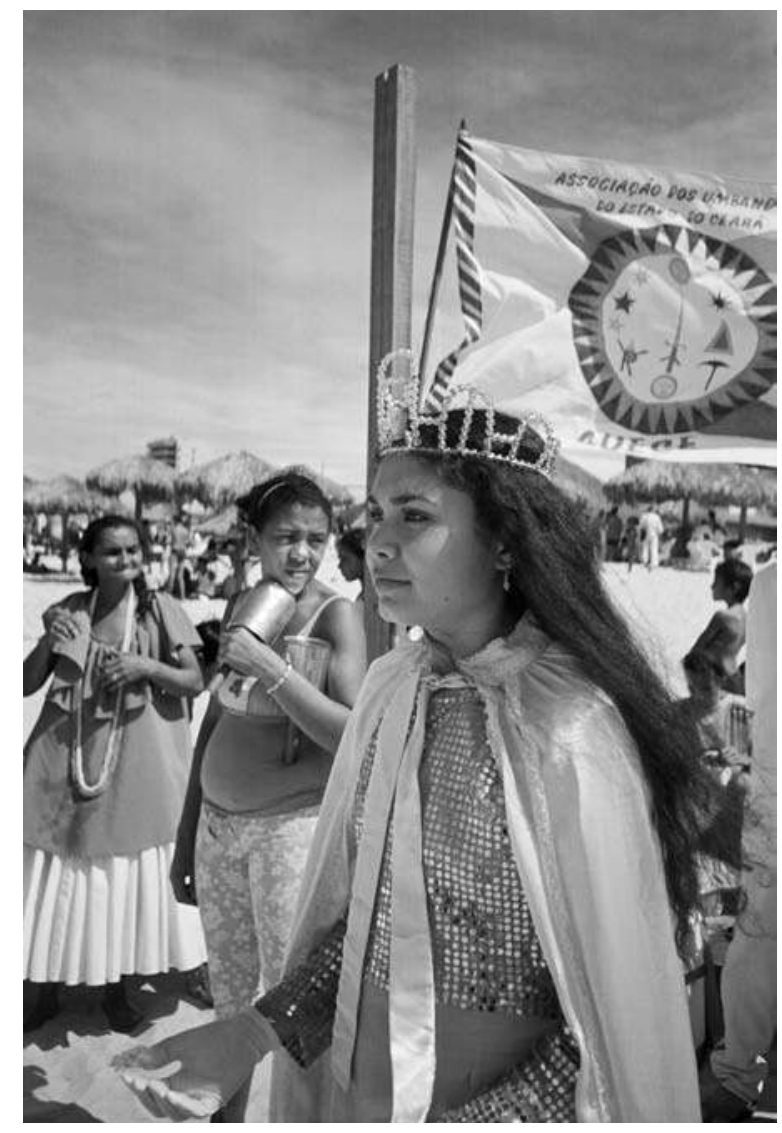

Fig. 7 Fille-de-saint portant les habits de lemanjá lors de la fête en l'honneur de lemanjá. Fortaleza, 2005. Photo I. P.

disgracieux que celui de João, elle qui était aussi belle et jeune qu'il était vieux et laid. Quand elle laissa apparaître ses traits, on put voir qu'elle avait outrageusement fardé la face de João.

La cérémonie prit alors la forme d'une sorte de show de cabaret, non au sens métaphorique que Leiris donne aux aspects théâtraux de la possession (1958), mais bien au sens premier du terme. La cigana commença par danser et chanter dans un cercle formé par les membres du terreiro, assis sur les chaises en plastique qui faisaient auparavant face à l'autel. Tout cela ressemblait fort à un spectacle de travestis, mais pourquoi être surpris? Lorsqu'elle était sur terre, la cigana était une artiste, une danseuse, une chanteuse, me dit-elle. Elle prit ensuite place sur l'une des chaises du cercle et entama une conversation mondaine en buvant du vin mousseux dans un verre à pied. Cette conversation ne manquait pas de piquant. La cigana n'est pas vulgaire comme Simbamba, elle s'efforce de séduire les hommes.

16. La cigana est une pomba gira, une exu féminine. Les pombas giras ne sont pas nécessairement noires ou métisses : la cigana est d'origine espagnole, tout comme une autre pomba gira fameuse du nom de Maria Padilha (Meyer 1993). 


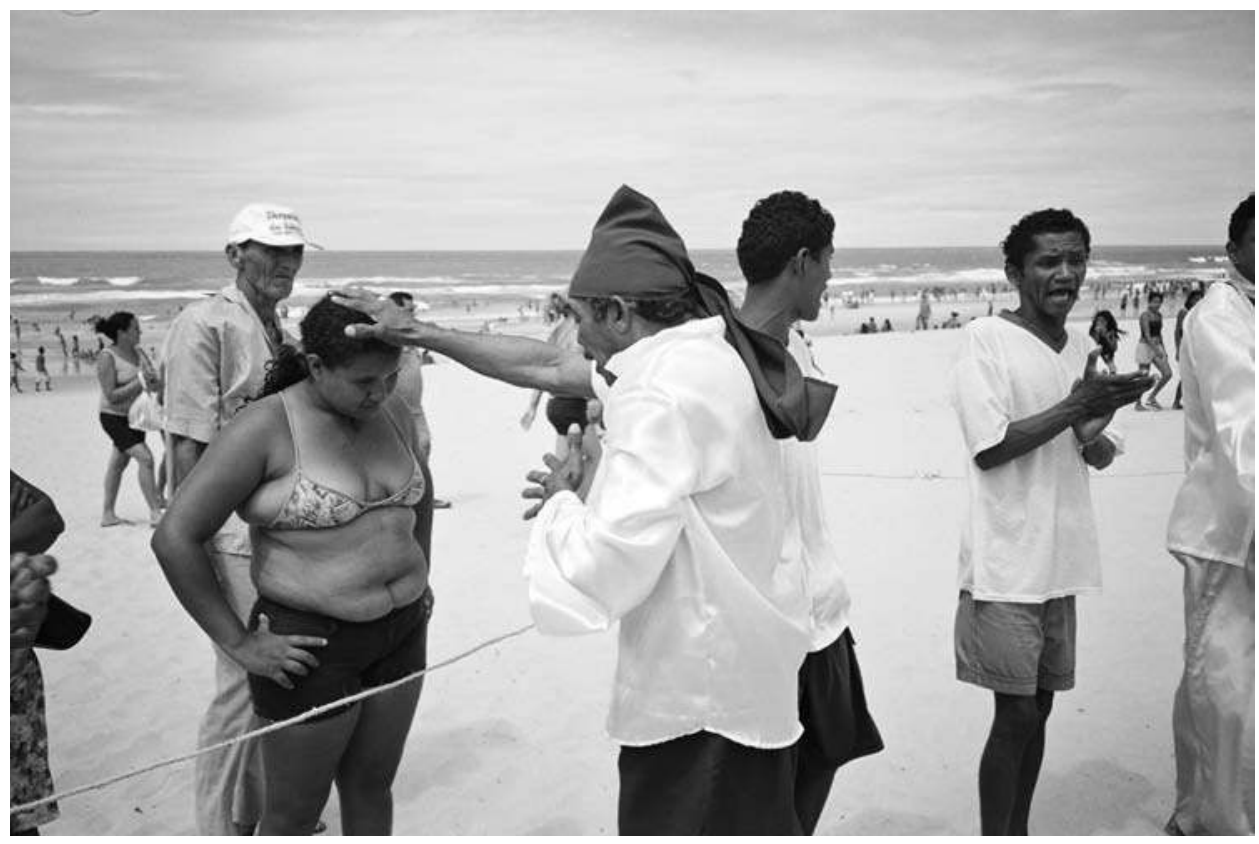

Fig. 8 Père-de-saint procédant à une guérison par imposition de main sur une participante à la fête pour lemanjá. Fortaleza, 2005. Photo I. P.

Elle donna des conseils en matière de cœur à plusieurs femmes de l'assistance, puis la façon dont elle devait s'y prendre pour partir avec moi à Paris en avion devint le principal thème de conversation. D'autres femmes s'en mêlèrent et demandèrent elles aussi à aller en France, mais la cigana le leur défendit formellement; la place était pour elle, et pour personne d'autre : «Qu'elles se trouvent un autre Français! "Elle me demanda alors de m'asseoir près d'elle afin d'avoir une petite conversation privée (audible par tous, bien entendu), dont le contenu était purement galant.

L'une de mes questions s'est néanmoins écartée de ce jeu plaisant : je demandais à la cigana comment elle avait vécu et comment elle était morte. Elle me dit qu'elle était une prostituée et qu'elle méritait de mourir. Je lui demandais pourquoi. Elle répondit sans sourciller, le regard fixe et le visage impassible, qu'elle avait avorté vingt-sept fois et que c'est pour cette raison qu'un homme l'avait poignardée vingt-sept fois. Certes, la mort violente est associée à l'image populaire de la gitane andalouse (c'est bien sûr la Carmen de Mérimée, qui meurt tragiquement assassinée par l'amant qu'elle a abandonné). Mais cette référence à l'avortement rappelle aussi le mythe d'Oya Yansan, connue comme divinité du Niger et de la tempête, que l'on nomme «mère des neuf » car elle eut neuf enfants. En Afrique, on l'invoque dans la divination par le jet de seize cauris en cas d'infertilité. Le signe Osa ${ }^{17}$ la présente effectivement comme une femme stérile rendue fertile par l'offrande d'un agneau et d'une pièce d'étoffe multicolore. Mais depuis qu'elle a mangé de l'agneau pour guérir sa stérilité, cette viande lui est interdite : c'est une nourriture taboue pour tous ceux qu'elle protège (Bascom 1980: 45,231 ). Ce jeu d'attirance et de répulsion est aussi au fondement de la relation de l'orixa avec ses enfants.

À Cuba, le rapport entre Oya et l'avortement est explicite, comme en témoigne Lydia Cabrera (1954:33) :

[J'ai été témoin] de la honte d'une femme qu'Oya, indignée, accusait publiquement d'avoir avorté plusieurs fois. Elle lui fit jurer « de ne pas jeter l'enfant qu'elle avait dans le ventre, sans quoi c'est elle que [la déesse] emporterait dans l'autre monde».

Plus loin, l'ethnographe cubaine rapporte le mythe suivant (ibid. : 97), qui témoigne d'une attitude beaucoup plus ambivalente de l'orixa à l'égard de sa progéniture :

Oya donna le jour [à Exu] et l'abandonna dans la savane. Les années passèrent, et Oya voulut le reconnaître comme son fils, mais il s'y refusa et lui jeta au visage qu'elle l'avait abandonné. [Un autre informateur déclare :] «Oya est une femme qui ne souhaite avoir ni maison ni enfant. Elle n'aime pas les enfants. »

Reste à établir le lien entre Oya et la gitane. À Fortaleza, c'est une pomba gira, c'est-à-dire un avatar féminin d'Exu, l'esprit du mauvais garçon dans l'um-

17. Le signe OSA correspond à neuf cauris « ouverts » et sept cauris « fermés » lorsque l'on jette le jeu de coquillages. 
banda, sans que le rapport entre la cigana et Oya, l'orixa de la tempête soit très clair ${ }^{18}$. Le détour par Cuba est ici encore fort utile : dans l'île des Caraïbes, Oya est représentée par une gitane, danseuse de flamenco, vêtue d'une robe multicolore à dominante écarlate comme le tissu offert par Oya pour enfanter (Dianteill 2000 : 120). Dans le candomblé, ses adeptes portent des colliers de couleur rouge grenat (Verger $1995: 166$ ); cette couleur a donc dû jouer un rôle dans le transfert de certains attributs de l'Oya du candomblé à la cigana de l'umbanda. Bref, la figure de Yansan, dont l'ambivalence à l'égard de l'enfantement est essentielle, apparaît en filigrane dans celle de la cigana, qui a avorté et qui a été poignardée vingt-sept fois, c'est-à-dire trois fois neuf, le chiffre d'Oya par excellence. Même si l'umbanda semble bien éloignée de la matrice mythique africaine, on voit que des paradigmes puissants issus de cette source, qui mettent en jeu le sexe, la mort et la génération, y sont toujours actifs.

À ce moment, l'assemblée se fit silencieuse. On était passé du sermon avec pai Roxo à la farce avec Simbamba, et enfin du vaudeville à la tragédie avec la cigana assassinée. Lorsque cette dernière annonça son départ, elle dit que d'autres esprits souhaitaient saluer le Français. Comment ne pas voir ici encore une allusion au théâtre, les acteurs saluant le public à la fin de la représentation? Ainsi, se succédèrent très rapidement dans le corps de João, sans parler, les entités suivantes : un preto velho, Joazinha (une criança, un esprit enfant) et un autre caboclo. Finalement, le dernier esprit quitta son corps pour s'envoler vers l'astral, et João reprit ses esprits (ou plutôt les perdit...) et revint à lui.

Les questions posées aux caboclos et à la pomba gira autant que ma présence dans le temple ont assurément modifié le déroulement habituel de la cérémonie. Pai Roxo, confronté à des questions de nature plus théologique que pratique, en a profité pour rendre compte des relations éthiques entre les hommes et les esprits. Simbamba a quant à lui détourné la situation à son profit en se moquant à la fois de l'étranger que j'étais, de la consultante de Rio de Janeiro au téléphone et, de façon plus détournée, du ton didactique et moralisateur qu'avait adopté pai Roxo plus tôt dans la soirée. Enfin, la cigana, face à des questions portant sur sa vie passée, a réintroduit dans la cérémonie un élément tragique hérité de la mythologie africaine. Dans les trois cas, l'irruption du chercheur contraint les acteurs de l'umbanda à s'adapter à un interlocuteur complètement étranger à leur monde, dont le discours et la présence mêmes sont incongrus.

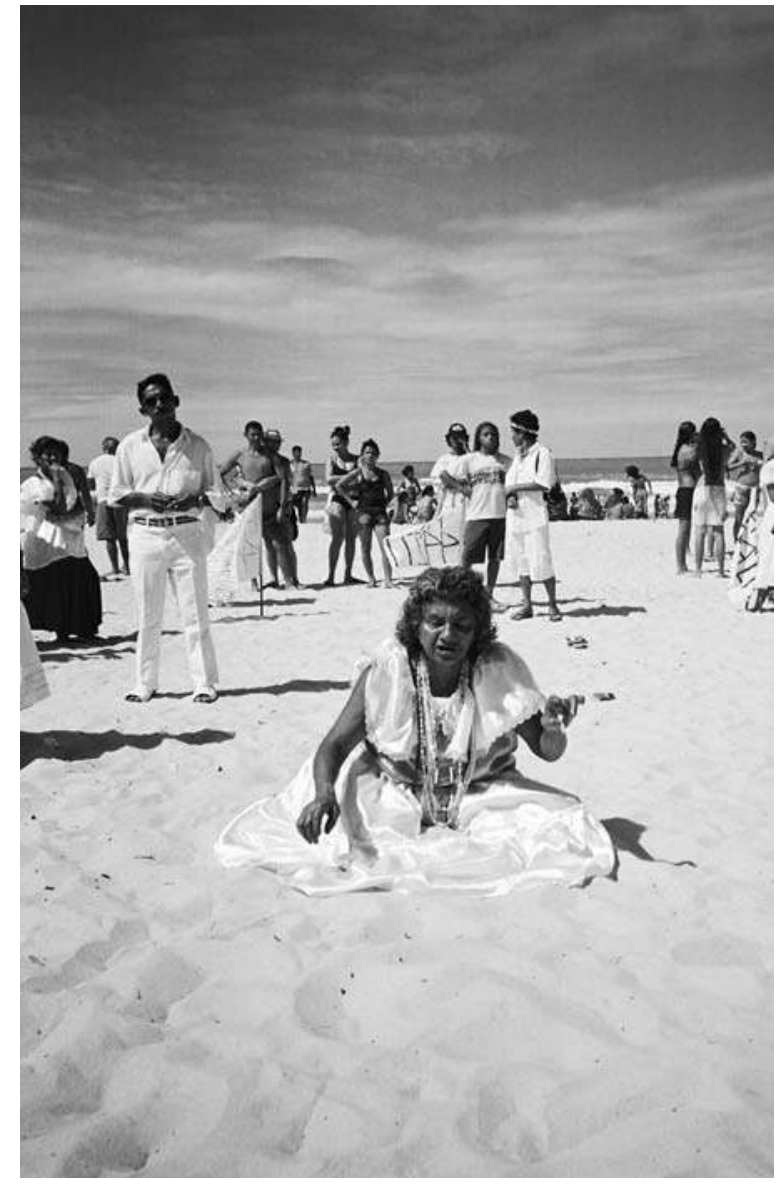

Fig. 9 Mère-de-saint en transe lors de la fête pour lemanjá. Fortaleza, 2005. Photo I. P.

\section{Conclusion : communication immatérielle} et royaume des esprits

L'histoire des religions afro-américaines est par définition une histoire de déterritorialisation et de reterritorialisation, au Brésil (Motta 2001) comme à Cuba (Dianteill 2002). Mais l'umbanda brésilienne redouble ce processus. Il ne s'agit pas d'une religion africaine déplacée, et plus ou moins transformée. Elle est pratiquée aujourd'hui par une majorité d'individus sans lien généalogique avec l'Afrique, et pour qui ce continent

18. Notons néanmoins qu'Oya est l'un des orixas les plus populaires dans la culture brésilienne. Jorge Amado en fait l'orixa éponyme de l'un des ses romans (Yansan des orages), et très récemment un dessin animé intitulé Yansan, de Carlos Eduardo Nogueira (2006), relate les relations tumultueuses de Yansan, Ogum et Xango, dans un style de manga japonais, sur un mode violent et érotique. Les éléments du mythe originel sont réinterprétés dans un contexte ultramoderne. Ogum, dieu du fer et de la forge, est un motard garagiste. Oya suit un traitement contre la stérilité grâce à une compagnie d'ingénierie génétique nommée Dollylnc, du nom de la brebis clonée, mais aussi référence à l'agneau sacrifié par l'orixa. Ce traitement lui permet d'accoucher de neuf enfants. 
n'est qu'une vague référence idéologique, parfois même sur un mode dépréciatif. Constituée dans le sud du Brésil à partir de sources africaines et spirites, l'umbanda a été introduite dans le Ceará dans les années 1950, dans une sorte de double déplacement entre le Sud industriel et le Nordeste. Mais, si les gens se déplacent, les esprits aussi; ils descendent du ciel pour intervenir dans les affaires humaines, interagissant avec une personne par le truchement d'un médium ou du téléphone. Cette intrusion peut prendre la forme d'une transgression : la traversée des frontières cosmologiques va souvent de pair avec des comportements incongrus, c'est-à-dire déplacés, comme celui de Simbamba. Qu'y a-t-il de plus invraisemblable que la situation suivante? Simbamba est un Indien mort, obsédé par les femmes, s'exprimant par la bouche d'un Blanc homosexuel et exigeant d'un Français qu'il dise des obscénités par téléphone à une personne qu'il n'a jamais vue, et qui se trouve à Rio, à près de trois mille kilomètres de là... Nous savons depuis Leiris (1958) et Métraux (1958) que toute forme de possession implique des aspects théâtraux (et souvent comiques). C'est aussi le cas de l'umbanda (Turner 1986), mais l'âge de la globalisation ajoute quelque chose à cette dramaturgie : il multiplie les possibilités de courtscircuits sociaux et symboliques, les courts-circuits de la pensée qui sont une source essentielle du rire selon Bastide (1970).

Je me suis efforcé ici de documenter brièvement les processus de transition entre le local, le global et le spatial dans le cas de l'umbanda. Mais ce que nous obser- vons à Fortaleza n'est pas si rare ailleurs. Les téléphones cellulaires rendent les consultations de voyance plus facile. C'est le cas pour les génies patros étudiés par Bertrand Hell à Mayotte, qui répondent au téléphone lorsqu'ils possèdent un médium, mais aussi de certains leaders d'Églises spirituelles à La Nouvelle-Orléans (Dianteill 2006), qui assurent des consultations téléphoniques inspirées par l'esprit Black Hawk avec des consultants appelant de Las Vegas ou de Los Angeles. Cette communication dématérialisée a assurément une affinité avec l'idée d'un cosmos rempli d'esprits désincarnés qui «flottent » dans l'« astral ». La communication instantanée, la réduction du temps et de l'espace caractérisent la sur-modernité selon Marc Augé (1992) ; ces traits sont aussi constitutifs d'une vision du monde où les esprits peuvent se déplacer instantanément d'un lieu à un autre, ou même être dans plusieurs lieux à la fois. N'est-ce pas ce que permettent le téléphone mobile et la vidéoconférence? Pour finir, n'oublions pas que notre présence même, en tant qu'anthropologues étrangers, signifie toujours l'entrée du monde dans un lieu particulier : même sans le vouloir, nous sommes aussi des vecteurs de globalisation.

mots clés / keywords : religions afro-brésiliennes // AfroBrazilian religions • Brésil // Brazil • umbanda // umbanda - globalisation // globalisation $•$ possession // possession.

CEIFR/EHESS edi@quaibranly.fr

\section{Bibliographie}

\author{
AMADO, Jorge \\ 1987 Yansan des orages. Paris, Gallimard. \\ AugÉ, Marc \\ 1992 Non-lieux. Introduction à une \\ anthropologie de la sur-modernité. Paris, Seuil. \\ BASCOM, William \\ 1980 Sixteen Cowries. Yoruba divination from \\ Africa to the New World. Bloomington et \\ Indianapolis, Indiana University Press. \\ BASTIDE, Roger \\ 1960 Les Religions africaines au Brésil. \\ Contribution à une sociologie des \\ interpénétrations de civilisation. Paris, PUF.
}

1970 « Le rire et les courts-circuits de la pensée », in Jean Pouillon et Pierre Maranda, [éd.], Mélanges offerts à Claude Lévi-Strauss à l'occasion de son $60^{e}$ anniversaire, t. Il. Paris-La Haye, Mouton : 953-963.

Brown, Diana De G. et Bick, Mario

1987 «Religion, Class and Context: Continuities and Discontinuities in Brazilian Umbanda », American Ethnologist 14[1] : 73-93.

CABRERA, Lydia

1954 El Monte. La Havane, ediciones C.R.

DianteILl, Erwan

2000 Des dieux et des signes. Initiation, écriture et divination dans les religions afro-cubaines. Paris, École des hautes études en sciences sociales.
$2002 \ll$ De-territorialisation and reterritorialisation of the Orisha religion in Africa and in the New World (Nigeria, Cuba, United States) », International Journal of Urban and Regional Research (Oxford, UK) 26[1] : 121-13?.

2006 La Samaritaine noire. Les Églises spirituelles noires américaines de La NouvelleOrléans. Paris, École des hautes études en sciences sociales [《Cahiers de L'Homme »).

DuvignaUd, Jean

2007 [1977] Le Don du rien. Paris, Téraèdre.

Falgayrette-Leveau, Christiane et Dianteill, Erwan (éd.)

2005 Brésil, l'héritage africain. Paris, Dapper. 
Giobelina BRumana, Fernando et Gonzalez Martinez, Elda

1989 Spirits in the Margin. Umbanda in São Paulo. Uppsala, Acta Universitatis UpsaliensisAlmqvist et Wiksell International.

\section{HALE, Lindsay Lauren}

1997 « Preto Velho: Resistance, Redemption and Endangered Representations of Slavery in a Brazilian Possession-Trance Religion 》, American Ethnologist 24(2) : 393-414.

\section{Hell, Bertrand}

1999 Possession et chamanisme. Les maîtres du désordre. Paris, Flammarion.

Hess, David J.

1989 «Disobsessing Disobsession: Religion, Ritual and the Social Sciences in Brazil », Cultural Anthropology 4[2] : 183-193.

1990 « Ghosts and Domestic Politics in Brazil: Some Parallels between Spirit Possession and Infestation 》, Ethos 18[4] : 407-438.

\section{LEIRIS, Michel}

1958 La Possession et ses aspects théâtraux chez les Éthiopiens de Gondar. Paris, Gallimard.

\section{Maggie, Yvonne}

2001 [1975] Guerra de Orixá. Um estudo de ritual e conflito. Rio de Janeiro, Jorge Zahar.

\section{MÉTrauX, Alfred}

1958 Le Vaudou haïtien. Paris, Gallimard.

Meyer, Marlyse

1993 Maria Padilha e toda a sua quadrilha: de amante de um rei de Castela a pomba-gira de Umbanda. São Paulo, EBC Nordeste-Livraria Duas Cidades.

\section{MotTA, Roberto}

2002 «L'expansion et la réinvention des religions afro-brésiliennes : réenchantement et décomposition », Archives de sciences sociales des religions 117 : 113-125.

2001 « Déterritorialisation, standardisation, diaspora et identités : à propos des religions afro-brésiliennes », in Jean-Pierre Bastian, Françoise Champion et Kathy Rousselet (éd.), La Globalisation du religieux. Paris,

L'Harmattan-Association française de sciences sociales des religions.

\section{Nogueira, Carlos Eduardo}

2006 Yansan (dessin animé), production Glaz Cinema, $18 \mathrm{mn}$.

\section{NsondÉ, Jean de Dieu}

1999 Parlons Kikôngo. Le lâri de Brazzaville et sa culture. Paris, L'Harmattan.

\begin{abstract}
OrTIZ, Renato
1978 A morte branca do feiticeiro negro. Umbanda: Intergração de uma religião numa sociedade de classes. Rio de Janeiro, Vozes.
\end{abstract}

\section{PORDEUS, Ismael Jr.}

2000 Magia e trabalho. A representação do trabalho na Macumba. São Paulo, Terceira margem.

2002 Umbanda. Ceará em transe. Fortaleza, Museu do Ceará.

\section{PRANDI, Reginaldo}

1995 «As religiões negras do Brasil. Para uma sociologia dos cultos afrobrasileiros », Revista USP 28 : 64-83.

\section{TURner, Victor}

1986 «Social Dramas in Brazilian Umbanda: The Dialectics of Meaning », in The Anthropology of Performance. New York, Performing Arts Journal Publications : 33-71.

VERGER, Pierre

1995 Dieux d'Afrique. Paris, Revue Noire.

\section{Résumé / Abstract}

Erwan Dianteill, Le caboclo surmoderne. Globalisation, possession et théâtre dans un temple d'umbanda à Fortaleza [Brésil]. - Cet article documente les processus de transition entre le local, le global et le spatial dans le cas de l'umbanda au Brésil, et en particulier dans le cas d'un temple de Fortaleza. Constituée dans le sud du Brésil à partir de sources africaines et spirites, l'umbanda a été introduite dans le Ceará dans les années 1950, dans une sorte de double déplacement entre le Sud industriel et le Nordeste. Si les gens se déplacent, les esprits aussi ; ils descendent du ciel pour intervenir dans les affaires humaines, en interagissant avec une personne par le truchement d'un médium ou par téléphone. Cette intrusion peut prendre la forme d'une transgression : la traversée des frontières cosmologiques va souvent de pair avec des comportements incongrus, c'est-à-dire déplacés, de certains esprits. La communication instantanée, la réduction du temps et de l'espace caractérisent la sur-modernité selon Marc Augé (1992) ; ces traits sont aussi constitutifs d'une vision du monde où les esprits peuvent se déplacer instantanément d'un lieu à un autre, ou même être dans plusieurs lieux à la fois.
Erwan Dianteill, The supermodern caboclo. Globalisation, possession and theatre in an Umbanda temple in Fortaleza [Brazil]. - This article documents the processes of transition between the local, the global and the spatial in the case of the Umbanda in Brazil, and specifically in a temple in Fortaleza. The Umbanda, which developed in southern Brazil from African and spiritualist origins, was introduced in the Ceará in the 1950s, in a sort of double movement between the industrial South and the Nordeste. If people moved, so did the spirits; they come down from the sky to intervene in human affairs, by interacting with a person through a medium or by telephone. This intrusion can take the form of a transgression: the crossing of cosmological frontiers often comes together with behaviour which is incongruous, that is to say, out of place, on the part of certain spirits. Instant communication, the reduction in time and space, characterise supermodernity according to Marc Augé; these characteristics are also constitutive of a vision of the world in which spirits can move instantaneously from one place to another, or can even be in several places at once. 